Romualdo Monteiro de Resende Costa

Controle do Sincronismo Temporal de Aplicações Hipermídia

Tese apresentada ao Programa de Pós-graduação em Informática da PUC-Rio como requisito parcial para obtenção do título de Doutor em Informática.

Orientador: Prof. Luiz Fernando Gomes Soares 
Romualdo Monteiro de Resende Costa

\section{Controle do Sincronismo Temporal de Aplicações Hipermídia}

Tese apresentada como requisito parcial para obtenção do grau de Doutor pelo Programa de Pósgraduação em Informática do Departamento de Informática do Centro Técnico Científico da PUCRio. Aprovada pela Comissão Examinadora abaixo assinada.

Prof. Luiz Fernando Gomes Soares Orientador

Departamento de Informática - PUC-Rio

Prof. Sérgio Colcher

Departamento de Informática - PUC-Rio

Prof. Simone Diniz Junqueira Barbosa Departamento de Informática - PUC-Rio

Prof. Cesar Augusto Camillo Teixeira Universidade Federal de São Carlos - UFSCar

Prof. Guido Lemos de Souza Filho Universidade Federal da Paraíba - UFPB

Prof. José Eugenio Leal Coordenador Setorial do Centro Técnico Científico 
Todos os direitos reservados. É proibida a reprodução total ou parcial do trabalho sem autorização da universidade, do autor e do orientador.

\section{Romualdo Monteiro de Resende Costa}

Graduou-se em Ciência da Computação pela UFMG em 2000. Obteve em 2005 o título de Mestre em Informática pela PUC-Rio. Desde 2002 é Oficial do Exército, na especialidade de informática. É pesquisador do Laboratório Telemídia da PUC-Rio.

Ficha Catalográfica

Costa, Romualdo Monteiro de Resende

Controle do sincronismo temporal de aplicações hipermídia / Romualdo Monteiro de Resende Costa; orientador: Luiz Fernando Gomes Soares. - 2010.

160 f. : il.(color.) ; $30 \mathrm{~cm}$

Tese (Doutorado) - Pontifícia Universidade Católica do Rio de Janeiro, Departamento de Informática, 2010.

Inclui bibliografia

1. Informática - Teses. 2. HTG. 3. TV digital. 4. Hipermídia. 5. Grafo temporal. 6. NCL. 7. Sincronismo. 8. Interatividade. I. Soares, Luiz Fernando Gomes. II. Pontifícia Universidade Católica do Rio de Janeiro. Departamento de Informática. III. Título. 
Este trabalho é dedicado

A Aline, pelo amor e dedicação.

A toda a minha família, pelo apoio e incentivos constantes.

E a Deus, por iluminar os nossos caminhos. 


\section{Agradecimentos}

Em especial, ao meu orientador, professor Luiz Fernando Gomes Soares, agradeço pela orientação, incentivo e disposição para acompanhar cada passo deste trabalho. Meus agradecimentos, no entanto, vão muito além dos ensinamentos necessários à elaboração deste trabalho. Obrigado pela amizade, pela confiança e pelo apoio em todos os momentos.

Aos amigos do Laboratório Telemídia, agradeço pela disposição de sempre cooperar e contribuir. Ao Moreno e ao Carlos, agradeço pelo exemplo que faz desse laboratório um ambiente de trabalho agradável e, ao mesmo tempo, muito produtivo. Ao Márcio, agradeço pelos trabalhos em conjunto e pelas contribuições necessárias à realização deste trabalho. Obrigado a todos os membros desse laboratório, incluindo aqueles que já integraram a nossa equipe, pela amizade e pelo convívio proporcionados ao longo desses anos.

Agradeço também ao Exército Brasileiro, representado pelos seus ilustres diretores: Gen Garcez, Cel Ferrari e TC Paulo, que compreenderam a importância deste trabalho e ofereceram o apoio necessário para que ele pudesse ser realizado.

Aos demais amigos agradeço pelo apoio, mesmo nas vezes em que me fiz ausente, tomado pelas atividades deste trabalho. Nesse período, tive a oportunidade de conferir que as verdadeiras amizades, ao contrário das aplicações hipermídia, são atemporais.

Agradeço a todos os professores e funcionários do Departamento de Informática, e à PUC-Rio, como instituição, pelo suporte financeiro. 


\section{Resumo}

Costa, Romualdo Monteiro de Resende; Soares, Luiz Fernando Gomes. Controle do Sincronismo Temporal de Aplicações Hipermídia. Rio de Janeiro, 2010. 160p. Tese de Doutorado - Departamento de Informática, Pontifícia Universidade Católica do Rio de Janeiro.

A preservação do sincronismo das aplicações hipermídia é um dos mais importantes requisitos de qualidade para uma apresentação. Para garantir essa qualidade, é necessário conhecer os instantes no tempo de ocorrência dos eventos existentes nas aplicações. Por meio dessa informação, é possível prever a ocorrência de atrasos e, mais do que isso, antecipar as ações necessárias à apresentação, com o objetivo de evitar a necessidade de ajustes provocados por esses atrasos. Esta tese discute como as ocorrências desses eventos podem ser previstas durante uma apresentação. Para auxiliar essa tarefa, um grafo temporal, construído a partir da especificação de uma aplicação, é proposto. Esse grafo, denominado HTG (Hypermedia Temporal Graph), pode ser utilizado no controle do sincronismo temporal de uma aplicação, desde o transporte das mídias até a sua apresentação nos clientes. Além da preservação da qualidade, esta tese explora outras vantagens que podem ser obtidas a partir do controle do sincronismo temporal das aplicações. Esse controle pode ser utilizado, por exemplo, para posicionar as apresentações em um instante qualquer no tempo, retrocedendo ou avançando até um ponto desejado. Outra vantagem relacionada a apresentação, também explorada nesta tese, é a distribuição de partes de uma aplicação para apresentação em diferentes dispositivos, sem que o sincronismo temporal de toda a aplicação seja prejudicado. Por fim, a atualização das aplicações, simultaneamente à sua apresentação, também é explorada nesta tese.

\section{Palavras-chave}

HTG; TV Digital; Hipermídia; Grafo Temporal; NCL; Sincronismo; Interatividade. 


\section{Abstract}

Costa, Romualdo Monteiro de Resende; Soares, Luiz Fernando Gomes. Temporal Synchronism Control of Hypermedia Applications. Rio de Janeiro, 2010. 160p. DSc. Thesis - Departamento de Informática, Pontifícia Universidade Católica do Rio de Janeiro.

Synchronization control in hypermedia applications is one of the most important requirements in the presentation of these applications. To assure highquality presentations, it is necessary to know when events, produced during an application execution, occur in time. By means of this information, it is possible to predict undesirable delays and to forestall actions needed to avoid presentation adjustments. This thesis discusses how event occurrences can be predicted in the course of an application presentation. In order to assist this goal, a temporal graph, built up from the application specification, is proposed. This graph, called Hypermedia Temporal Graph - HTG, can be used in the temporal control of hypermedia applications, from the media transport system up to their presentation at client sides. Besides the quality of service control, this thesis elucidates other advantages that come from the synchronization management. Among them is allowing for presentations to be started at any moment in time of their life cycle, and allowing for presentations to be moved backward and forward up to a desirable presentation moment in time. Another advantage, also dealt with in this thesis, is the support to distributing parts of an application to different devices in charge of their presentations, without causing any hazard to the whole application temporal synchronism. Finally, this thesis also proposes how editions can be made over the HTG, and, therefore, how application control can be modified during runtime.

\section{Keywords}

HTG; Digital TV; Hypermedia; Temporal Graph; NCL; Synchronism; Interactivity; 


\section{Sumário}

1 Introdução 15

1.1. Motivação 16

1.1.1. Controle do Tempo da Apresentação e da Transmissão 16

1.1.2. Edição Simultânea à Apresentação 18

1.1.3. Apresentação Distribuída através de Múltiplos Dispositivos 19

1.2. Objetivos 20

1.3. Organização da Tese $\quad 21$

2 Trabalhos Relacionados $\quad 23$

2.1. Controle do Tempo da Apresentação e Transmissão 24

2.2. Edição Simultânea à Apresentação 29

2.3. Apresentação Distribuída através de Múltiplos Dispositivos 31

3 Hypermedia Temporal Graph - HTG 34

3.1. Modelo de Grafos Temporais 34

3.1.1. Definição 34

3.1.2. Exemplo 37

3.1.3. Construção 43

3.2. Cadeias Temporais $\quad 47$

4 Representação de Aplicações Hipermídia Declarativas Aplicações NCL 56

4.1. Módulos, Perfis e Aplicações NCL 56

4.2. Objetos de Mídia e Interfaces 59

4.3. Adaptação da Apresentação e Adaptação do Conteúdo 62

4.4. Reúso 65

4.5. Relacionamentos 69

4.6. Contextos 76

5 Planos para o Controle do Sincronismo Temporal 86

5.1. Planos para o Controle da Execução de uma Aplicação 86 
5.2. Planos para o Transporte e Carregamento do Conteúdo

5.2.1. Controle da Distribuição do Conteúdo

5.2.2. Controle do Carregamento do Conteúdo

5.3. Controle do Sincronismo Temporal no Ginga-NCL para

TV Terrestre

6 Edição e Distribuição de Apresentações Hipermídia

6.1. Edição Simultânea à Apresentação 115

6.1.1. Edição ao Vivo nas Aplicações NCL 116

6.1.2. Edição ao Vivo no HTG 117

6.2. Apresentação Distribuída através de Múltiplos Dispositivos 122

7 Conclusões 129

7.1. Contribuições da Tese 129

7.2. Trabalhos Futuros 130

8 Referências 132

Apêndice A - Sincronismo Temporal de Aplicações Hipermídia 140

A.1. Modelos de Sincronização Temporal 141

A.1.1. Sincronização Baseada em um Eixo do Tempo (Timeline) 141

A.1.2. Sincronização Hierárquica 144

A.1.3. Sincronização Baseada em Redes de Petri 146

A.1.4. Sincronização Baseada em Causalidade e/ou

Restrição de Eventos 149

A.1.5. Sincronização Baseada em Grafos Temporais 150

Apêndice B - Comandos de Edição 154 


\section{Lista de figuras}

Figura 3.1 - Máquina de estado de um evento.

Figura 3.2 - Visões espaciais da aplicação exemplo

(primeira parte).

Figura 3.3 - Visões espaciais da aplicação exemplo

(segunda parte).

39

Figura 3.4 - HTG para a aplicação apresentada

nas Figuras 3.2 e 3.3.

40

Figura 3.5 - Cadeia temporal principal do HTG apresentado

na Figura 3.4.

51

Figura 3.6 - Cadeias temporais secundárias do HTG da Figura 3.4.

51

Figura 3.7 - HTG com vários eventos imprevisíveis.

Figura 3.8 - Cadeia temporal principal do HTG

da Figura 3.7.

Figura 3.9 - Duas cadeias temporais secundárias do HTG

da Figura 3.7.

Figura 3.10 - Cadeia HTG resultante da junção da cadeia principal com a primeira cadeia secundária.

Figura 3.11 - Um possível HTG final para a apresentação da Figura 3.4.

Figura 4.1 - Apresentação de uma aplicação NCL

com interatividade.

Figura 4.2 - Objetos de mídia e suas interfaces na aplicação NCL exemplo.

Figura 4.3 - Parte do HTG que representa os objetos

e suas interfaces descritos na Figura 4.2.

Figura 4.4 - Adaptação da apresentação na aplicação

NCL exemplo.

Figura 4.5 - Parte do HTG que representa a adaptação da apresentação descrita na Figura 4.4.

Figura 4.6 - Adaptação do conteúdo na aplicação NCL exemplo. 
Figura 4.7 - Parte do HTG que representa a adaptação do conteúdo descrita na Figura 4.6. 64

Figura 4.8 - Reúso na aplicação NCL exemplo. 66

Figura 4.9 - HTG para o exemplo de reuso "gradSame". 68

Figura 4.10 - Base de conectores utilizados na aplicação

NCL exemplo. $\quad 69$

Figura 4.11 - Relacionamentos associados ao sincronismo

no tempo (sem interação) na aplicação NCL exemplo. 71

Figura 4.12 - Parte do HTG que representa o sincronismo

no tempo especificado na Figura 4.11.

72

Figura 4.13 - Relacionamento associado ao sincronismo

no tempo (com condição) na aplicação NCL exemplo.

73

Figura 4.14 - Parte do HTG que representa o sincronismo

no tempo (com condição) especificado na Figura 4.13.

Figura 4.15 - Relacionamento associado ao sincronismo

no tempo (com interação) na aplicação NCL exemplo.

75

Figura 4.16 - Parte do HTG que representa o sincronismo no tempo (com interação) especificado na Figura 4.15.

Figura 4.17 - Relacionamento associado a um evento

de atribuição na aplicação NCL exemplo.

Figura 4.18 - Parte do HTG que representa o evento

de atribuição especificado na Figura 4.17.

Figura 4.19 - Visão estrutural de um contexto com ações.

Figura 4.20 - Parte de um HTG que representa as ações de "start", "stop" e "abort" sobre o contexto da Figura 4.19.

Figura 4.21 - Parte de um HTG que representa a ação de

"pause" sobre o contexto da Figura 4.19.

80

Figura 4.22 - Parte de um HTG que representa a ação de "resume" sobre o contexto da Figura 4.19.

Figura 4.23 - Visão estrutural de um contexto com condições associadas.

Figura 4.24 - Parte do HTG que representa as condições "onBegin", "onPause" e "onEnd" sobre o contexto da Figura 4.23. 
Figura 4.26 - HTG correspondente à aplicação NCL

da Figura 4.25.

Figura 5.1 - Cadeia temporal principal do HTG construído

no Capítulo 4.

Figura 5.2 - Cadeias temporais secundárias do HTG construído no Capítulo 4.

Figura 5.3 - HTG final para a especificação temporal descrita na Tabela 5.16.

Figura 5.4 - Arquitetura Ginga: módulos relacionados ao controle do sincronismo no Ginga-NCL.

Figura 5.5 - Diagrama de pacotes referente aos componentes do HTG.

Figura 5.6 - Descrição do componente Planos HTG.

Figura 5.7 - Tempos para o carregamento do Ginga-NCL para o conjunto de aplicações da Tabela 5.19.

Figura 5.8 - Menu LUA do Ginga-NCL para acesso ao plano de apresentação.

Figura 5.9 - Cadeias HTG construídas pelo componente Planos HTG para uma aplicação NCL (Aplicação 3 da Tabela 5.19).

Figura 6.1 - Múltiplos dispositivos para exibição.

Figura 6.2 - HTG para controle da apresentação nos dispositivos individuais.

Figura 6.3 - Distribuição de dispositivos em domínios.

Figura A.1 - Exemplo de sincronização hierárquica. 


\section{Lista de tabelas}

Tabela 3.1 - Regras para a construção do HTG.

Tabela 3.2 - Regras para a especificação de cadeias HTG. 49

Tabela 4.1 - Principais características relacionadas ao sincronismo e à adaptação encontradas na aplicação NCL exemplo.

Tabela 4.2 - Valores reservados para papéis utilizados como condição.

70

Tabela 4.3 - Valores reservados para papéis utilizados como ação.

Tabela 4.4 - Comportamento dos componentes de um contexto de acordo com as ações realizadas sobre esse contexto. 78 Tabela 4.5 - Estados da apresentação de um contexto. 81 Tabela 5.1 - Especificação temporal da cadeia principal. Tabela 5.2 - Especificação temporal da cadeia iniciada no Vértice 6.

Tabela 5.3 - Especificação temporal da cadeia iniciada no Vértice 8.

Tabela 5.4 - Especificação temporal da cadeia iniciada no Vértice 9.

Tabela 5.5 - Especificação temporal da cadeia iniciada no Vértice 12.

Tabela 5.6 - Especificação temporal da cadeia iniciada no Vértice 13.

Tabela 5.7 - Especificação temporal da cadeia iniciada no Vértice 14.

Tabela 5.8 - Especificação temporal da cadeia iniciada no Vértice 16.

Tabela 5.9 - Especificação temporal da cadeia iniciada no Vértice 19.

Tabela 5.10 - Especificação temporal da cadeia iniciada no Vértice 21. 
Tabela 5.11 - Especificação temporal inicial do plano de apresentação.

Tabela 5.12 - Especificação temporal do plano de apresentação modificado (1).

Tabela 5.13 - Especificação temporal do plano de apresentação modificado (2).

Tabela 5.14 - Especificação temporal do plano de apresentação modificado (3).

Tabela 5.15 - Especificação temporal do plano de apresentação modificado (4).

Tabela 5.11 - Estados alcançáveis através da máquina de estado de eventos.

Tabela 5.16: Especificação temporal final do plano

de apresentação.

Tabela 5.17 - Especificação temporal final do plano de carregamento de exibidores para as cadeias das Figuras 5.1 e 5.2.

Tabela 5.18 - Especificação do plano de distribuição para as cadeias das Figuras 5.1 e 5.2 .

Tabela 5.19 - Informações do HTG para um conjunto de aplicações.

Tabela 6.1 - Comandos para inserir, remover ou editar vértices e arestas que representam um evento de apresentação ou a adaptação do conteúdo.

Tabela 6.2 - Comandos para inserir, remover ou editar condições nas arestas que representam a adaptação do conteúdo ou da apresentação.

Tabela 6.3 - Comandos para inserir, remover ou editar arestas que representam a adaptação da apresentação.

Tabela 6.4 - Comandos para inserir, remover ou editar vértices e arestas que representam aos relacionamentos de uma apresentação.

Tabela B.1 - Comandos de edição.

Tabela B.2 - Identificadores utilizados nos comandos de edição. 